\title{
Preoperative Three-dimensional Virtual Simulation for Safe Liver Surgery
}

Tetsuya Shimizu ${ }^{1}$, Nobuhiko Taniai ${ }^{1}$, Masato Yoshioka ${ }^{1}$, Hideyuki Takata ${ }^{1}$, Tomohiro Kanda ${ }^{1}$, Yoshiaki Mizuguchi ${ }^{1}$,

Yasuhiro Mamada ${ }^{1}$, Hiroshi Yoshida ${ }^{2}$ and Eiji Uchida ${ }^{1}$

${ }^{1}$ Department of Surgery, Nippon Medical School

${ }^{2}$ Department of Surgery, Nippon Medical School Tama Nagayama Hospital

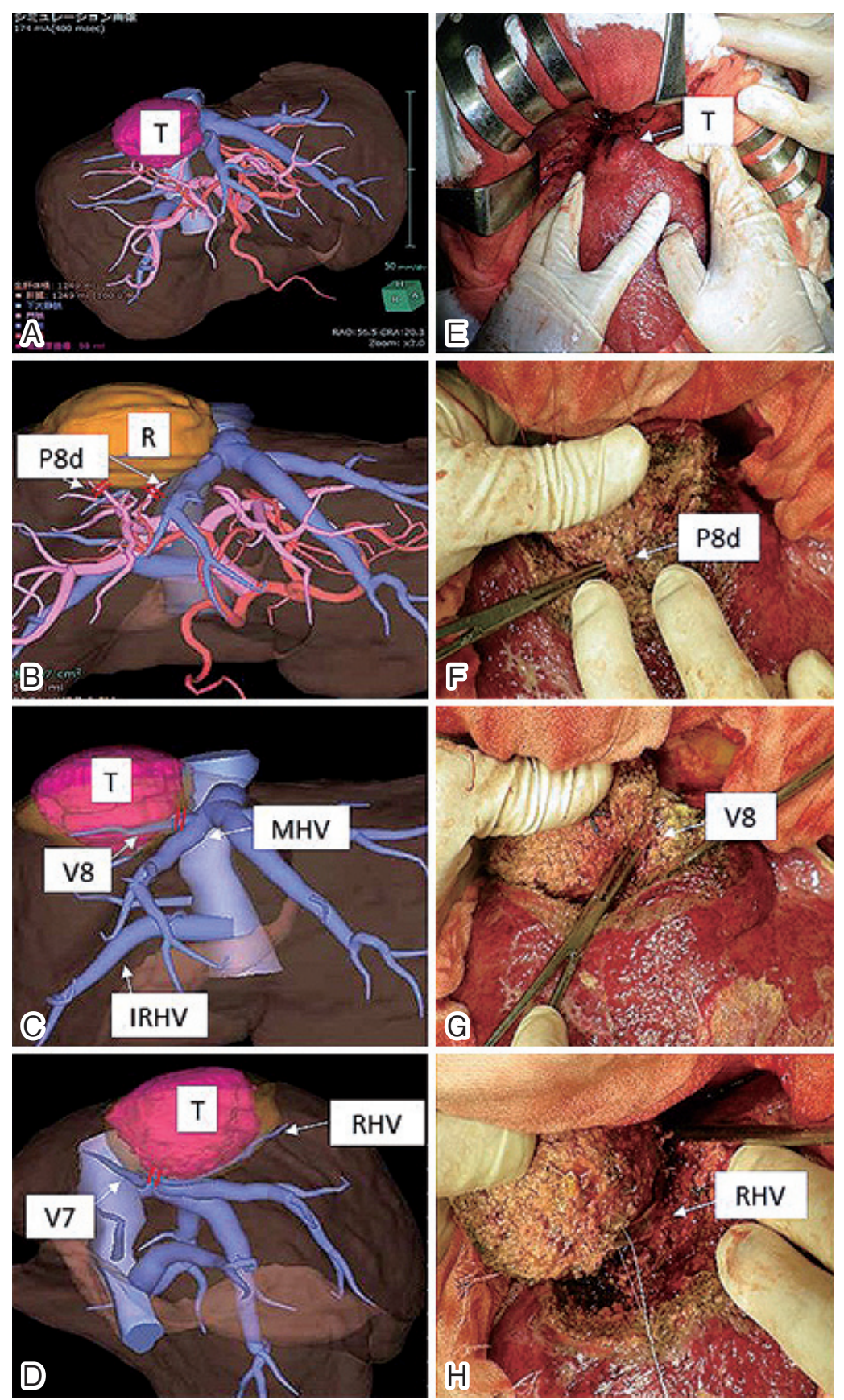

Fig. 1

Correspondence to Tetsuya Shimizu, MD, Department of Surgery, Nippon Medical School, 1-1-5 Sendagi, Bunkyo-ku, Tokyo 113-8603, Japan 
The recent progress of image analysis technology has been remarkable, and preoperative virtual simulation for liver surgery is necessary for safety hepatectomy ${ }^{1.2}$. It has become easy to grasp the complicated anatomical relationship between the portal triad, hepatic veins and a local tumor by volume rendering with multidetectorrow computed tomography and a three-dimensional image analysis workstation (Synapse Vincent ${ }^{\circledR}$; Fuji Photo Film Co. Ltd.).

In patients with damaged liver function or extended hepatectomies, the postoperative residual liver volume with preservation of blood supply and drainage vessels is very important for the prevention of liver failure. In this software, the surgeon can simulate various patterns of planned hepatectomy.

\section{Case}

A 79-year old female was referred for resection of a hepatocellular carcinoma. She suffered from chronic coughing and decreased respiratory function due to interstitial pneumonia with liver dysfunction due to chronic hepatitis C.

CT revealed a peripherally enhanced low-density mass $5.0 \mathrm{~cm}$ in diameter located in Segment VIII/VII. In consideration of her comorbidities, we simulated a safe liver resection using Vincent ${ }^{\circledR}$. Figure 1A-D show the preoperative simulation of the hepatectomy. Figure 1A displays the perspective of the liver and the tumor. The tumor is located in segment VIII/VII just under the right diaphragm. In Figure 1B, two portal branches of the dorsal portion of segment VIII (P8d) were identified as main feeders of the resected liver area. The position of the tumor relative to a drainage vein in segment VIII (V8) and the right hepatic vein (RHV) are shown in Figure $\mathbf{~}$ C and D. The distal portions of the V8 and the RHV after divergence from the middle hepatic vein (MHV) and the V7, respectively, were invaded by the tumor, and the V8 and the RHV needed to be resected. However, the MHV and the V7 were not directly influenced by the tumor, and the MHV and the V7 were thought to be able to be preserved. In consideration of these simulation images and her comorbidities, we selected a partial resection of segment VIII/VII with preservation of the MHV and the V7 as a recommended safe operation. Figure 1E-H show images from the operation. Figure 1E shows the local presence of the tumor and the liver according to the simulation image. Two P8ds were accepted according to the simulation of the liver excision line (Fig. 1E). The V8 (Fig. 1G) and the RHV (Fig. 1H) with invasion by the tumor were also identified during the hepatectomy and separated after ligation. The V7 and the MHV were confirmed to be preserved by intraoperative ultrasonography.

Conflict of Interest: The authors declare no conflict of interest.

Fig. 1 Preoperative three-dimensional virtual simulations (A-D) and images from the operation corresponding to these simulation images $(\mathrm{E}-\mathrm{H})$.

T: tumor, R: resected liver area, P8d: portal segment branches of the dorsal portion of segment VIII, V8: drainage vein of Segment VIII, MHV: middle hepatic vein, RHV: right hepatic vein, IRHV: inferior right hepatic vein

\section{References}

1. Mochizuki K, Takatsuki M, Soyama A, et al: The usefulness of a high-speed 3D-image analysis system in pediatric living donor liver transplantation. Ann Transplant 2012; 17: 31-34.

2. Itoh S, Shirabe K, Taketomi A, et al.: Zero mortality in more than 300 hepatic resections: validity of preoperative volumetric analysis. Surg Today 2012; 42: 435-440.

E-mail: tetsuya@nms.ac.jpＪournal Website (http://www.nms.ac.jp/jnms/) 